\title{
PAULUS PEZZUOLO
}

alumnus Scholae Normalis Superioris,

Piazza dei Cavalieri 7, 56126 Pisis

Italia

\section{DE GENERE DICENDI DELIBERATIVO A LATRONIBVS APULEIANIS VSVRPATO (MET. VI, VII)}

\begin{abstract}
Pezzuolo Paulus, De genere dicendi deliberativo a latronibvs Apuleianis vsvrpato (Met. VI, VII) (O doradczym rodzaju wypowiedzi zastosowanym przez rozbójników u Apulejusza (Met. VI, VII)).

In books VI and VII of Apuleius' Metamorphoses we find the robbers delivering some orations, which imitate the genus deliberativum: they display sollemnity and refined elegance to such an extent, that the reader, being aware that they proceed from the most heinous rogue's lips, cannot but smile by himself.

Composing this part of his tale, Apuleius also falls back on and re-treats some elements of the greek novel, in particular the representation of the band of robbers like a sort of perverted state.

In this article I will first show the resemblances that Apuleius' rogue-tales share with the same places of the Greek novels' writers, then I will continue to examine the speeches found in books VI and VII.

I will demonstrate with how much elaboration Apuleius has amplified and augmented these tales, which the Greek novelists have merely outlined, for the sake of playing on extant literature, and I will clarify to what extent we can understand this playing on literature as a parody.
\end{abstract}

Keywords: robbers, greek novel, genus deliberativum, Apuleius, Metamorphoses, anti-society, comic.

Quisquis partem illam Metamorphoseon evolvit, in qua Apuleius facta $\mathrm{Lu}-$ cii a latronibus rapti prosequitur horumque res gestas refert (Met. IV, VI, VII), vel prima specie animadvertet has omnes fabulas singulari quodam artificio compositas esse; nam tota earum vis in eo consistit, quod furta, compilationes, omnia postremo perditorum hominum crimina ornatissima illustrantur oratione, et ea quidem, qualis vel in Caesaris encomio redundaret. Quae quidem sublimitas orationis complurium virorum doctorum studia excitavit, adeo ut nostra memoria multae existant symbolae ac commentationes, quibus genus dicendi Apuleianum, quale his narrationibus exarandis adhibitum sit, philologa ratione examinetur atque excutiatur, praecipue quod ad narrationem de mortibus Lamachi, Alcimi, Thrasyleonis spectat: haec enim cum copiis orandi' ${ }^{1}$, tum etiam

${ }^{1}$ Quod ad sermonem militarem spectat, multa quidem adnotata sunt ab expositoribus Groningianis (GCA 1977), adiecto ad hoc indice omnium locutionum (pp. 208-209) de imitatione 
inventorum novitate ceteris praenitet ${ }^{2}$. Itaque, cum inter viros doctos alius aliam provinciam explorandam sibi elegisset, indeque ex plurimis artificiis Apuleianis haud pauca ante latentia detecta sint, nonnulla vero, quae iampridem animadversa fuissent, in medio posita sint diligentiusque illustrata, illud inter omnes satis conveniet, in fabulis istis diversa genera dicendi adhiberi eaque sublimiora. Nam, si attenderis, sermonem militarem et philosophicum iuxta invenies, verba poetica et prisca cum lingua iuris consultorum temperata, imitationem tandem cum rhetorum et oratorum, tum etiam clarissimi cuiusque rerum scriptoris. Hac nimirum ratione oratio erigitur, sed tamen, cum in facta tam humilia cadat, risum etiam lectori excutit.

In hac autem symbola illud nobis proposuimus, ut quoad facultas ferret rationem illustraremus, qua Apuleius sermonem civilem usurpavit: quemadmodum videlicet verba ex senatu, conciliis, comitiis deducta in hanc partem operis sui transtulerit. Quae si accuratius investigare cupimus, quaeramus oportet quo modo haec ipsa argumenta - narrationes de latronibus videlicet- in fabulis Milesiis, unde Apuleius materiem derivavit, tractata sint.

Nam satis constat scriptores fabularum Graecarum, eos saltem quorum opera usque ad nostram memoriam pervenerunt, non minimum locum latronibus tribuisse: in illis praeterea, si animum intenderimus, animadvertemus latrones maxima ex parte certa atque communi ratione depingi. Nam tam in Charitonis quam in Xenophontis libris latrones, alterutrum amantium rapientes longe $\mathrm{ab}$ altero abducunt itaque efficiunt, ut eorum errores fiant longiores antequam ipsi iterum conveniant inter se; apud Heliodorum grex Thymaidis Memphitae

sermonis philosophici subtilissime disputatum est a Michaele Loporcaro in symbola a. 1992 edita (Loporcaro 1992), in qua inter alia mors Lamachi, quae in met. I 11 narratur, cum schematis luminibusque orationis contendit, quae veteres scriptores in excessibus virorum illustrium, praesertim philosophorum, describendis usurpaverint (haud pauca praeterea adnotantur de colore stoico istius loci); quae vero Ioannes Carolus Gianotti de imitatione sermonis iuridicialis coarguit (Gianotti 1981) mihi longe petita et a natura fabulae nostrae sat aliena videntur; quantum ad genus dicendi historicum, aliquid invenies in commentariis Groningianis; dictiones aliquae poeticae illustratae sunt a Catharina Lazzarini in symbola a. 1985 foras data (Lazzarini 1985). De colore epico diversae fuerunt sententiae A. La Penna et Staurus Frangoulidis, maxime de eo loco, quo Thrasyleon in aedes Democharis immissus equum Troianum innuere videatur: hic enim contendit hic inesse imitationem carminis heroi (Frangoulidis 1991), ille negat (La Penna 1985). Quod tandem aliquibus in locis tragici quiddam in hac fabula recinit, cum in verbis (Thebae heptapyli) tum etiam in rebus ipsis (facta Chryserotis), Harrison eo adductus est ut huic parti ludum quendam de tragoediis atticis assignaret (Harrison 2013: 203-205). Quod attinet ad ceteras de latronibus fabulas, multa reperies in expositione Larae Nicolini a. 2000 foras data (Nicolini 2000).

${ }^{2} \mathrm{Hac}$ nempe rerum novitate adductus P.A. Mackkay (Mackkay 1963) affirmavit materiem harum fabularum alieniorem esse a consuetudinibus scriptorum Graecorum quam ut a fabulis Milesiis derivata esse credatur: haec omnia esse nimirum a carminibus quibusdam Africis deducta, quae Apuleius Madauris audivisset. Ego ex contrario sat mihi persuadeo argumentum fabulae nostrae esse sincere Graecum: novitas universae narrationis oritur ex singulari Apuleii ingenio, qui materiem Graecam ita amplificavit immutavitque ut aliquid omnino novi effecisse videatur. 
causa est discidii ipsius; Apuleius, quamvis excepto commercio inter Photidem et Lucium nullum locum amoribus tribuat, latrones eodem fere consilio adhibet, scilicet ut errores Lucii producat.

Quodsi genus horum latronum eorumque societatis subtilius definire quaerimus, haud alienum erit verba Hendrici Hobsbawm referre, quae in libro de latronibus inveniuntur:

There is even a sharper division between peasant bandits and the criminal underworld of urban or vagant elements, which existed in the intersices of the rural society but clearly did not belong to it. In traditional societies criminals are almost by definition outsiders, who form their own separate society, if not actually an anti-society of the "bent" which mirrors that of the "straight". They normally speak their own special language (argot, cant, calo, Rotwelsch). Their association are with other outcast occupations or communities, like the gypsies [...] (The bulk of peasant bandits speak no kind of argot, but simply a version of the local peasant dialect) They are non- conformists, or rather anti-conformists in practice and by ideology, on the devil's side rather by God's, or if religious, then on the side of heresy against orthodoxy ${ }^{3}$.

Etenim tam in fabulis Milesiis quam apud Apuleium latrones sua instituta habent, sua sacra procurant, ritus proprios observant, ita ut in universum genere quodam perverso rei publicae frui videantur. Hoc ipsum, ut infra exhibebimus, ansam cum scriptoribus Graecis tum etiam Apuleio praebet ad schemata generis deliberativi affectanda. In huius videlicet opera ea, quae apud illos nonnisi adumbrate tractabantur, eo consilio amplificantur immo exaggerrantur, ut lector ad risum moveatur.

\section{RES PUBLICA LATRONUM}

Prius vero quam nodum huius symbolae aggrediamur, breviter ostendemus quibus maxime in rebus ritus horum latronum ab institutis iustarum civitatum discrepent. Qua de re tres saltem loci memorandi sunt.

a) De Martis cultu. Religio Martis fuit certe res satis celebrata a scriptoribus fabularum Milesiarum quotiescumque latrones inducendi erant. Etenim in Ephesiacis Xenophon induxit latrones Marti litantes hoc loco, in quo parum abest quin Anthia ipsa deo immoletur:

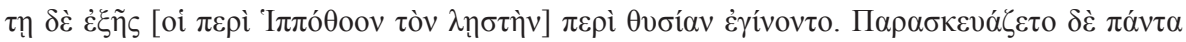

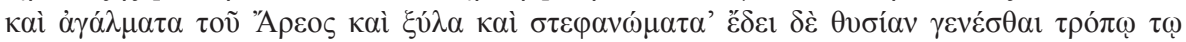

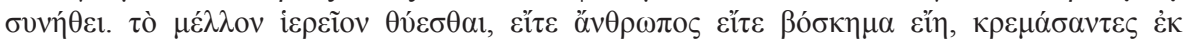

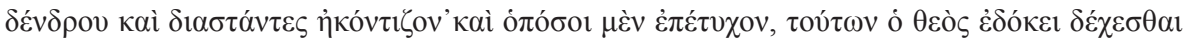

${ }^{3}$ Hobsbawm 1981: 39. 


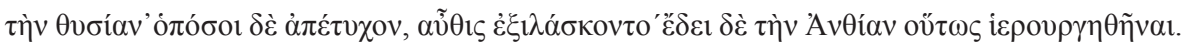
Eph. II. 13. 1

Haec apud Apuleium multo copiosius reperiuntur: in fabula de interitu trium latronum Lamachus iam moriturus commilitones obtestatur per fidem sacramenti atque per dexteram Martis (IV, 10); paulo inferius, latrones antequam in honorem commilitonum propinent deo Marti blandiuntur (IV, 22). Etiam in libris posterioribus mentio huiusmodi caerimoniarum invenitur: latrones enim appellantur tum Martia cohors, tum Marti clientes; paulo post ara in honorem Martis secutoris construitur suadente Haemo (Met. VII 11). Ad hoc sunt qui hanc caerimoniam desertorum condicioni attribuant.

b) De exsequiis. Eo loco ubi mortem Lamachi describit, Apuleius mentionem facit miri cuiusdam exsequiarum generis, quale nusquam apud veteres scriptores invenitur, quodque haud secus quam cultus Martis ad propria latronum instituta referendum videtur et ad illam perversam iustae civitatis imitationem de qua supra disputavimus. Commilitones enim Lamachi, ut in Met. IV 11 legitur, postquam eius corpus rite prosecuti sunt linteoque obvolverunt, in mare coniciunt:

Tunc nos magnanimi ducis vigore venerato corpus reliquum veste lintea diligenter convolutum mari celandum commisimus. Et nunc iacet noster Lamachus elemento toto sepultus.

Quo quidem in funere Lamachus singulari quodam honore affici videtur. Hoc quidem non potest quin admirationem nostram suscitet, cum alias cadaverum

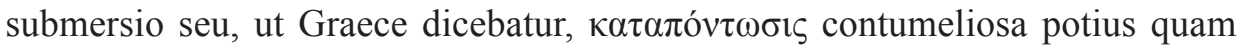
honorifica haberetur. Qua de re nobis succurrat, praeter plurimos eosdemque vulgatissimos locos quos scriptores et Graeci et Latini de cura corporum sepeliendorum habent, illa Chaereae oratio quam Chariton in primo libro fabularum suarum posuit. Illic enim adulescens, dum ipse se tamquam uxoris occisorem accusat, postulat a Syracusanorum senatu ut, postquam supplicium de se sumpserint, cadaver mari submergant (Charit. I 5)4. Mare enim Romanis Graecisque, quamvis minus quam aliis quibusdam gentibus, semper sinistrum visum est atque impurum. De his rebus probabiliter disseruit Petrus Janni in symbola dudum Urvini edita ${ }^{5}$, ubi praeterea illud de fabula nostra adnotat, tale genus funeris, quo latrones Lamachi corpus prosequuntur, propterea ut decorum atque honestum perhiberi, quod latrones mores perversos usquequaque prae se ferunt ${ }^{6}$.

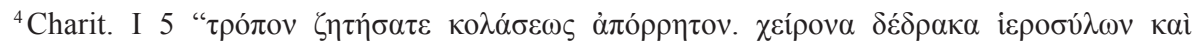

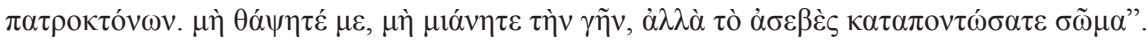

${ }^{5}$ Janni 2005.

${ }^{6}$ Cf. Janni 2005: 132: Qui tutte le valutazioni morali (e implicitamente religiose) sono rovesciate: i feroci banditi diventano personaggi epici e modelli di eroismo militare, pieni di orgogliosa fedelta al loro re...; ibid. 134: Ma in questa fittizia luce di eroismo e nobiltà, in questo grotte-
} 
c) De consilio latronum. Venio demum ad tertium latronum institutum, hoc est ad eorum concilia. Certis enim temporibus latrones consultationes instituunt, maxime cum de capite eius, quem rapuerint, deliberandum est. Hic est locus, ut monstrabimus, cum apud scriptores Milesios celebratissimus, tum ab Apuleio conscienter resumptus eoque consilio retractatus, ut nonnullis partibus auctis atque amplificatis, de hac re lusus quasi fieret risusque lectoribus exprimeretur. Hoc argumentum, cum iam in fabulis Charitonis inveniatur, tum multo auctius et illustrius in primo libro Heliodori repetitum videmus, ubi Thyamis latro de hac re ad commilitones refert, utrum fas esse arbitrentur Charicliam, puellam nuper raptam, in manum suam convenire. Singulos locos referentes eosdemque quantum poterimus illustrantes illud ostendere conabimur, quanta illis insit generis deliberativi imitatio. Hoc enim pacto fidenter ad nucleum huius commentationis promovebimur: proposuimus enim exhibere quo artificio Apuleius hoc ipsum genus dicendi in Metamorphosibus suis assimulaverit.

Apud Charitonem in libro primo comites Theronis, postquam Callirhoen cum divitiis ex sepulchro extraxerunt, deliberant quid tali in tempore sibi agendum sit, reddendane scilicet femina parentibus, an potius occidenda an- id quod tandem praevalet- cum ceteris spoliis in Graeciam aut Asiam deportanda ibique vendenda. Varietas mentium et consiliorum tum exhibetur:

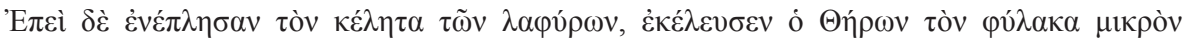

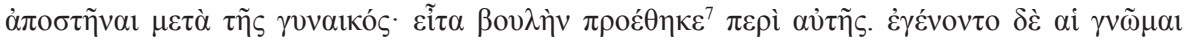

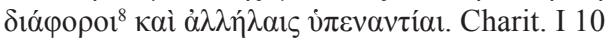

Primus latro, quippe qui callidior atque in fraudem proclivior sit, dolum in Callirhoae parentes machinatur:

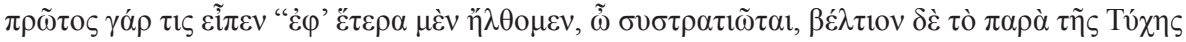

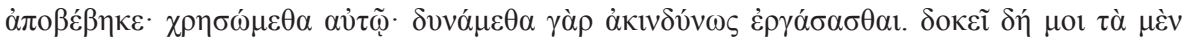

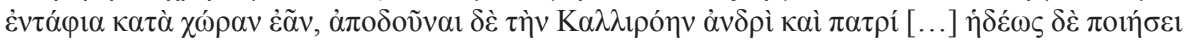

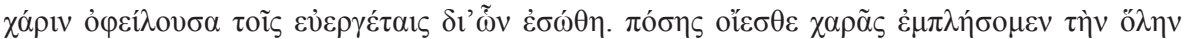

sco rovesciamento di ogni prospettiva consueta, diventa rispettabile e gloriosa anche una fine da carogna di animale, come doveva apparire alla mentalità antica.

${ }^{7} \mathrm{Haec}$ significatio verbi $\pi \rho \tau_{\imath} \theta \dot{\varepsilon} v \alpha 1$, h.e. ad senatum, ad populum de aliqua re referre, apud multos Graecos scriptores invenitur, praesertim ubi sequitur $\gamma v \omega ́ \mu \eta \nu$ (Thuc. I 132; VI 14) aut

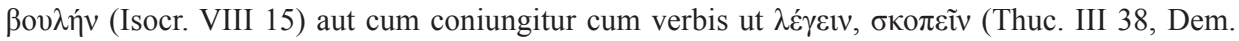
XVIII 273).

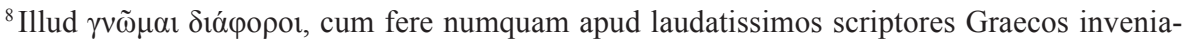
tur, tum haud raro in operibus christianorum reperies, fortassis ad exemplum locutionis Latinae

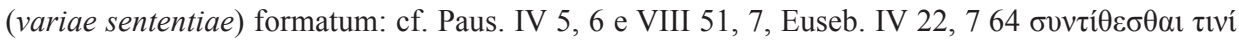
apud bonos Graecitatis auctores plerum significat cum aliquo pacisci, pangere, ut...; interdum autem idem signifcat atque apud Charitonem, h.e. alicui assentiri: cf. Lys. fr. 68, Call. Epigr. I 14, Hippol. Haer. VIII 19, 3. 




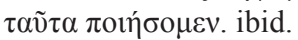

Alter contra, ferocior quidem neque tam subtilis, cum securitatem dolis praeferat, tum spissiores machinationes aspernari videtur:

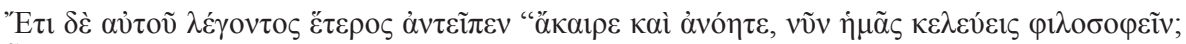

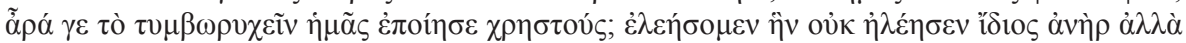

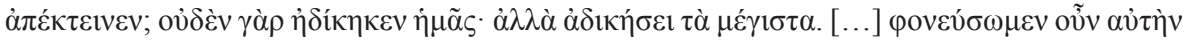

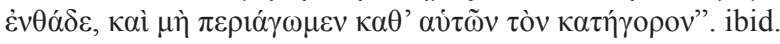

Theron ad extremum illud statuit quod per commune commodum fieri arbitratur:

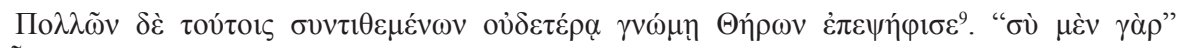

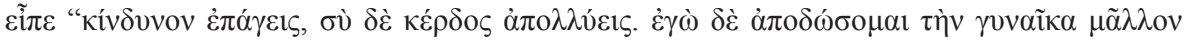

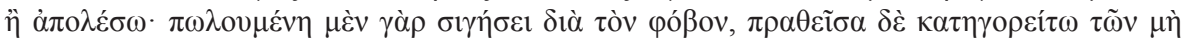

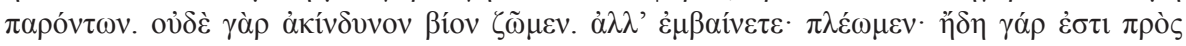

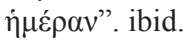

Latrones variorum ingeniorum esse, comprobatur etiam eo, quo Theron, compilationem sepulchreti nondum adortus, virtutes hominum suorum secum recenset:

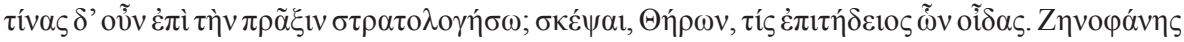

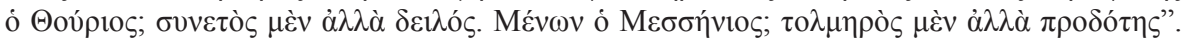

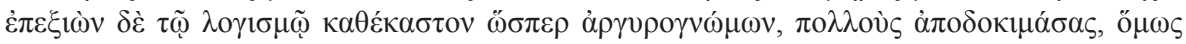

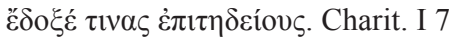

In libris Xenophontis Ephesii quamquam nihil huiusce modi invenitur, tamen, quoniam satis constat eos libros nihil esse nisi longioris fabulae epitomen, ego non longe absum quin credam fabulam pristinam tale aliquid habuisse.

Heliodorus autem in Aethiopicis suis simile quiddam depingit, multoque copiosius quam ceteri singulas res illustrat. In libro primo paludes describit, quas latrones eorumque socii accolunt haud procul a litore. Ibi autem omnia administrantur ut non ut a semiferorum hominum caterva, sed quasi ab hominibus certis legibus adstrictis, immo qui veluti parvam rem publicam constituisse videntur:

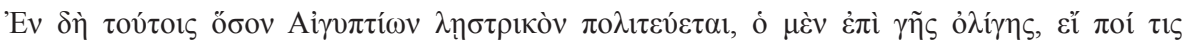

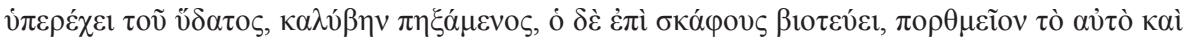

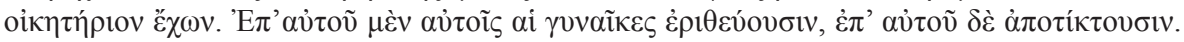

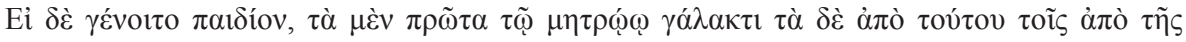

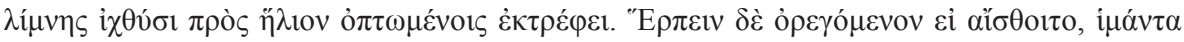

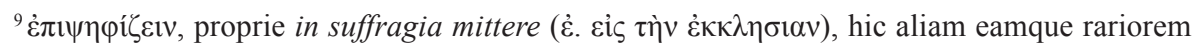

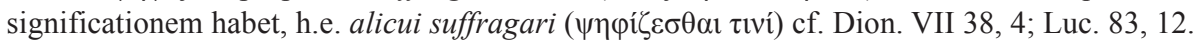




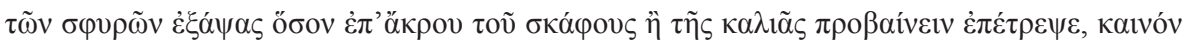

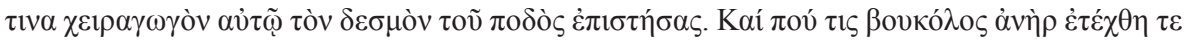

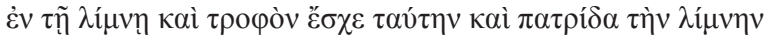

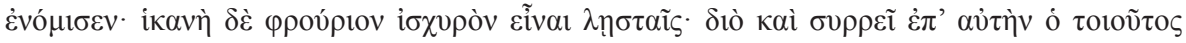

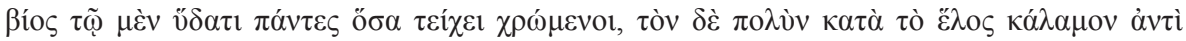

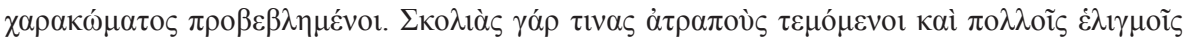

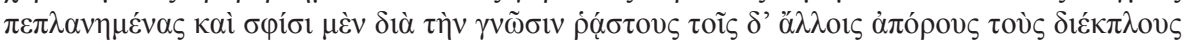

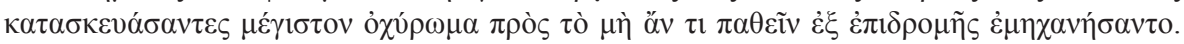
Heliod. Aeth. I, 7

Thyamis, utpote huius societatis princeps, domum occupat in insula a ceteris seiuncta, in qua una cum paucis spectatae fidei habitat, quasi imperator in aula:

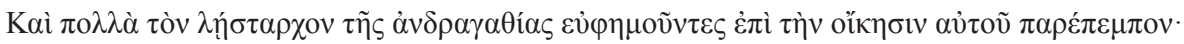

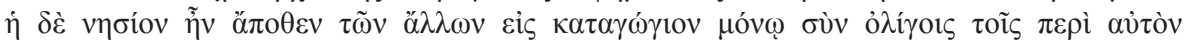
$\dot{\alpha} \pi \mathrm{o} \varepsilon \varepsilon \tau \mu \eta \mu \dot{\varepsilon}$ ov. ibid.

Singularis dignitas, qua Thyamis ornatur, tum vel magis animadvertitur, cum latrones in consilium coguntur, eumque audiunt orantem; cui quidem nihil oratoriae copiae abesse credas, adeo diserte, copiose, affluenter loquitur.

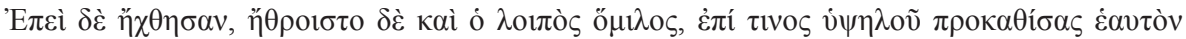

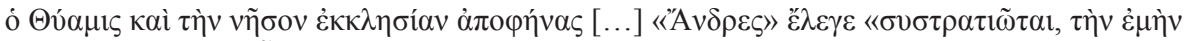

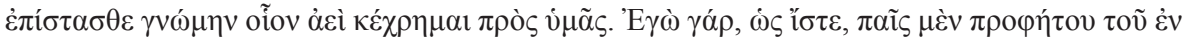

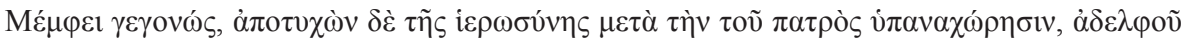

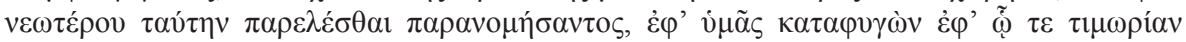

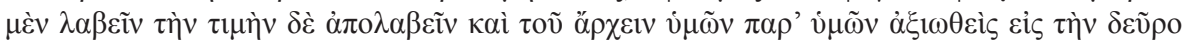

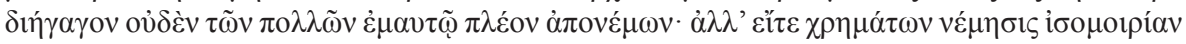

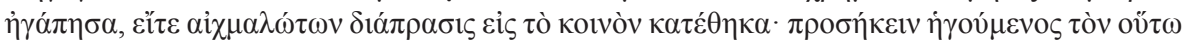

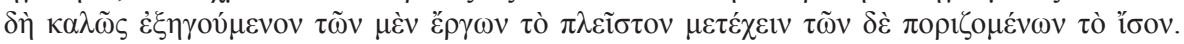
Heliod. Aeth. I 19-20

Praeter allocutionem ( $\left.\alpha{ }^{\prime} \delta \rho \varepsilon \varsigma \sigma v \sigma \tau \rho \alpha \tau \imath \tilde{\omega} \tau \alpha \imath\right)$ quae non sine sale eundem locum laudatissimorum oratorum Atheniensium redolet, animadvertenda est mirabilis omnium sententiarum gravitas et cauta argumentorum dispositio: primum enim fidem suam et bonam mentem erga commilitones commemorat, deinde aliqua subicit cum de maioribus suis, tum de dignitate, quam commilitonum suorum optione obtinet, postremo, ut eorum favorem captet, abstinentiae ac singularis aequitatis, quibus in regendo latronum coetu usus sit, mentionem facit. Post autem quam plura docuit de rationibus suis, tandem ad rem fidentius provehitur:

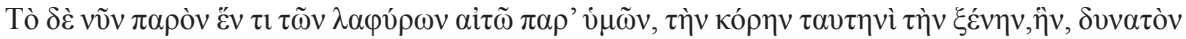

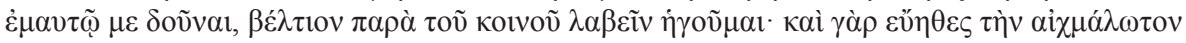

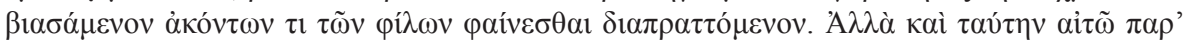

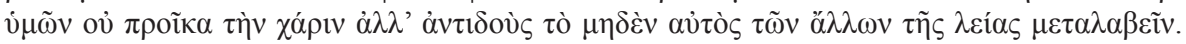

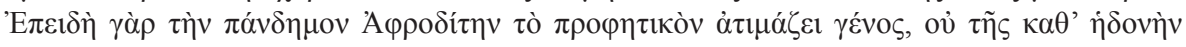

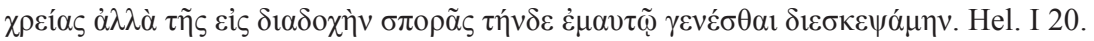


Multa deinceps verba de utilitate ac decore talis coniugii facit quoad, in fine loquendi, ita perorat:

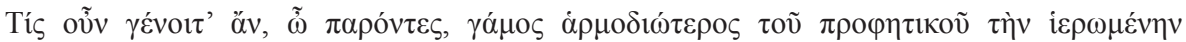

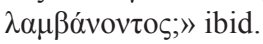

Tum vero, latronibus laudantibus proposita, ipse talia addit, quibus humanitatem suam omnibus probet:

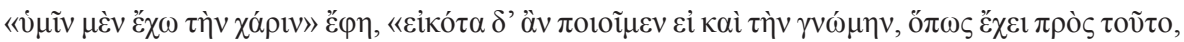

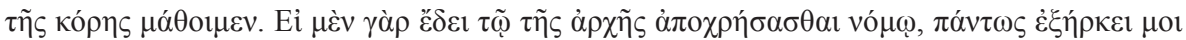

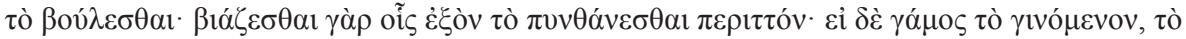

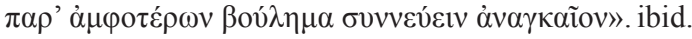

Chariclea autem, sententiam rogata, talem coram latronibus orationem instituit, ut vere videatur non in barbarorum coetu, sed in ipso foro Atheniensium versari:

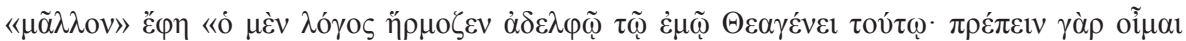

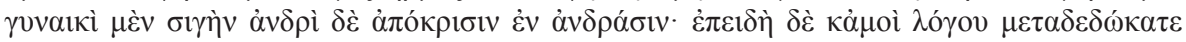

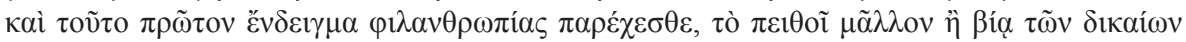

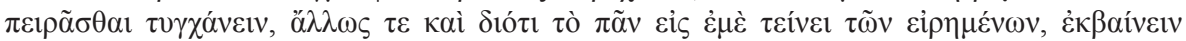

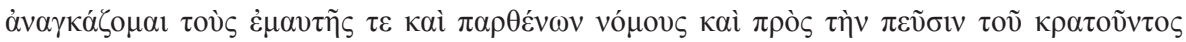

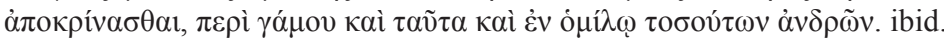

Hoc loco adeo discrepat tempus a verborum dignitate, a decore sententiarum, ab ipsa tandem complexionum magnitudine, ut ipsa orationis sublimitas paulo absurdior exsistat. Quin immo mihi facile persuadeo hic nonnullum locum risui datum esse, quamquam cetera fabula ab Heliodoro non nisi severe atque graviter tractatur; apud Apuleium autem, ut mox videbimus, iocandi voluptas totam fabulam, de qua hic agimus, informat.

Quod vero ad verba, quae quis propria generis deliberativi dixerit, apud Cha-

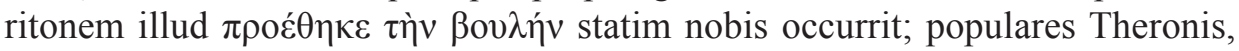

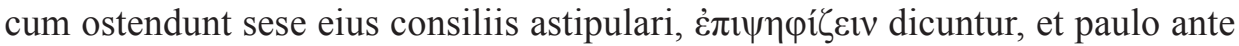
$\sigma v v \tau i ́ \theta \varepsilon \sigma \theta \alpha 1$; cum autem nova res initur legitur multum distant illa Apuleii ergo placuit ad hunc primum ferremus aditum (Met. IV 9).

\section{ORATIONES DE CAPITE CHARITES}

Haec omnia quae in fabulis Graecanicis agnovimus, plenius atque absolutius in libris medianis Metamorphoseon tractata esse videmus: et iam ad rem, quam enodare proposueram, venio. Consilia enim latronum Apuleius plura in Metamorphosibus collocavit, idque maxime in libro sexto et septimo ubi, postquam 
Charite e fuga retracta una cum asino in speluncam redigitur, latrones congregantur de poena, qua fugitivi plectendi sint, deliberaturi.

Cena igitur apposita hanc rem variis iactant sententiis:

Ac dum avida voracitate cuncta contruncant, iam incipiunt de nostra poena suaque vindicta secum considerare. Et utpote in coetu turbulento variae fuere sententiae, ut primus vivam cremari censeret puellam, secundus bestiis obici suaderet, tertius patibulo suffigi iuberet, quartus tormentis excarnificari praeciperet; certe calculo cunctorum utcumque mors ei fuerat destinata. Met. VI 30

Hic igitur, non secus quam in fabulis Graecanicis quas supra consideravimus, latrones in consilium coguntur ut de capite obsidum disputent: ab illis proferuntur variae sententiae ( $=\gamma v \tilde{\omega} \mu \alpha \mathrm{l} \delta$ ió $\varphi$ opol, Charit. I 10), omnesque locutiones, quibus deliberatio describitur, ex lingua senatusconsultorum exprompta sunt: censere, suadere, iubere, praecipere. Huc additur illud maxime deliberativum, cum Lucius memorat extremum supplicium Charitae intentari cunctorum calculo.

His dictis intercedit quintus latro qui ad consilium omnium crudelissimum comites suos impellit:

Tunc unus, omnium sedato tumultu, placido sermone sic orsus est: "Nec sectae collegii nec mansuetudini singulorum ac ne meae quidem modestiae congruit pati vos ultra modum delictique saevire terminum nec feras nec cruces nec ignes nec tormenta ac ne mortis quidem maturatae festinas tenebras accersere. Meis itaque consiliis auscultantes vitam puellae, sed quam meretur, largimini”. ibid. 31

In suadendo nihil est optabilius quam dignitas, monet Cicero in libris de oratore $^{10}$ : cui praecepto latro noster sedulo paret - nullum enim omittit dicendi artificium, quo orationem suam graviorem reddere possit atque ad senatoriam quandam severitatem efferre. Prooemium membra crescentia habet:

Nec sectae collegii

Nec mansuetudini singulorum

Ac ne meae quidem modestiae congruit...
Nec cruces

Nec ignes

Nec tormenta

Ac ne mortis quidem maturatae

festinas tenebras arcessere

In quo duo adnotanda videntur: primum orationis copia, quae haud secus atque in consilio Thyamidis, mirum in modum a feritate latronum dissonat risumque lectori aliquatenus exprimit; deinde abundantia dictionum civilium, quas Apuleius certe ludendi causa affectavit.

Nec vos memoria deseruit utique quid iam dudum decreveritis de isto asino semper pigro quidem sed manducone summo nunc etiam mendaci fictae debilitatis et virginalis fugae sequestro ministroque. Hunc igitur iugulare crastino placeat totisque vacuefacto praecordiis per

${ }^{10} \mathrm{Cic}$. de orat. II 233 
mediam alvum nudam virginem, quam praetulit nobis, insuere, ut sola facie praeminente ceterum corpus puellae nexu ferino coerceat, tunc super aliquod saxum scruposum insiciatum et fartilem asinum exponere et solis ardentis vaporibus tradere Sic enim cuncta quae recte statuistis ambo sustinebunt, et mortem asinus quam pridem meruit, et illa morsus ferarum, cum vermes membra laniabunt, et ignis flagrantiam, cum sol nimiis caloribus inflammarit uterum, et patibuli cruciatum, cum canes et vultures intima protrahent viscera. Sed et ceteras eius aerumnas et tormenta numerate: mortuae bestiae ipsa vivens ventrem habitabit, tum faetore nimio nares aestuabit, et inediae diutinae letali fame tabescet, nec suis saltem liberis manibus mortem sibi fabricare poterit. ibid. 32

Quod ad locutiones senatorias spectat haec illustriora sunt: nec vos memoria deseruit, quod etsi primum ab Apuleio hac iunctura expressum est, refert tamen ciceronianam illam locutionem memoria aliquem deficit ${ }^{11}$; huc adde placeat illud deliberandi gratia prolatum, verbum decernere, quod magis senatorium videtur quam instituere, constituere et similia; praeterea illud etiam vitam largimi$n i$, quod supra rettulimus, iudiciale aliquid recinere videtur, cum verbum largiri saepe usurpetur quoties de poena remittenda agitur ${ }^{12}$.

At si de gravitatis affectatione in universum loquendum est, nihil tantum ad simultationem dignitatis confert quam longitudo ipsa sententiarum perplexique circuitus. Hoc insuper adnotandum est, quod latro gregem appellant tum sectam, tum collegium: quae quidem vocabula, cum sacerdotum, philosophorum, iuris consultorum consessus plerumque designare soleant, hic potius maiestatis assimulandae gratia usurpantur- istud autem artificium non tantum hac in oratione observamus, sed in omnibus fere Metamorphoseon partibus in quibus latrones de se ipsis loquentes inducuntur ${ }^{13}$.

Affectatio sermonis curialis vel apertius apparet secundum finem orationis, ubi legimus:

talibus dictis non pedibus sed totis animis latrones in eius vadunt sententiam. ibid. 32

Haec omnia, quae in libro sexto demonstrata sunt, in septimo vel magis liquent, cum Haemi sequester res Hypatae gestas refert:

Is in primo speluncae aditu residens et ex anhelitu recepto spiritu tale collegio suo nuntium fecit: "Quod ad domum Milonis Hypatini quam proxime diripuimus pertinet, discussa sollicitudine iam possumus esse securi. Postquam vos enim fortissimis viribus cunctis ablatis castra nostra remeastis, immixtus ego turbelis popularium....". Met. VII, 1

Praeter alia quae etiam supra exhibuimus, hic observamus quem ad modum latrones nonnulla ex sermone castrensi mutuentur, cum v.g. latibulum nomine castrorum designatur ${ }^{14}$.

${ }^{11}$ Cic. inv. 112; Cic. Cat. 9.

${ }^{12}$ Cic. Cluent. 3; Caecin. 13.

${ }^{13}$ Met. IV 15, 1; VI 31; VII 4, 20; VII 7, 18.

${ }^{14} \mathrm{De}$ generibus dicendi quae in hac parte fabulae Apuleianae a latronibus usurpantur nonnulla adnotantur a Lara Nicolini (Nicolini 2000, passim), quae commentatio mihi quidem multo 
His igitur relatis Lucius aliquid subicit de sorte, quam sibi impendere videt:

Talibus cogitationibus fluctuantem subit me cura illa potior, qua statuto consilio latronum virginis decretam me victimam recordabar, ventremque crebro suspiciens meum iam misellam puellam parturibam. ibid. 4

In quibus dictis animadvertenda sunt illud statuto consilio latronum atque illud decretam me victimam, quae etiam verba curiam et forum quodammodo redolent.

Postea Tlepolemus, rebus suis expositis, a latronibus dux consalutatur.

Nec mora nec cunctatio, sed calculis omnibus ducatum latrones unanimes ei deferunt... sic reformatus, singulos exosculatus et in summo pulvinari locatus cena poculisque magnis inauguratur. ibid. 9

Post autem, cum sermo de poena Lucio Charitaeque intentata exoritur, Haemus alteram instituit oratiunculam, qua latrones a tam saevo consilio avertat. Operae pretium est totum exemplum huic commentationi inicere:

\begin{abstract}
"Non sum quidem tam brutus vel certe temerarius «inquit» ut scitum vestrum inhibeam, sed malae conscientiae reatum intra me sustinebo si quod bonum mihi videtur dissimulavero. Sed prius fiduciam vestri causa sollicito mihi tribuite, cum praesertim vobis, si sententia haec mea displicuerit, liceat rursus ad asinum redire. Nam ego arbitror latrones, quique eorum recte sapiunt, nihil anteferre lucro suo debere ac ne ipsam quidem saepe et ultis damnosam ultionem. Ergo igitur, si perdiderit in asino virginem, nihil amplius quam sine ullo compendio indignationem vestram exercueritis. Quin ego censeo deducendam eam ad quampiam civitatem ibique venundandam. Nec enim levi pretio distrahi poterit talis aetatula. Nam et ipse quosdam lenones pridem cognitos habeo, quorum poterit unus magnis equidem talentis, ut arbitror, puellam istam praestinare condigne natalibus suis fornicem processuram nec in similem fugam discursuram, non nihil etiam, cum lupanari servierit, vindictae vobis depensuram. Hanc et animo quidem meo sententiam conducibilem protuli; sed vos vestrorum estis consiliorum rerumque domini”. ibid. 9
\end{abstract}

In hac oratione omnia sunt ad suasorii generis rationes composita atque accommodata. Tlepolemus enim, qui simulat se in ea causa esse, ut aeque peccata Charitae iudicare possit, in exordio captationem benevolentiae praetendit, qua monstrat se aliter quidem sentire de puellae supplicio, nolle tamen libertatem latronum sua nova auctoritate opprimere: cum praesertim vobis, si haec mea sententia displicuerit, liceat ad asinum redire. Haec praefatus, admonet nihil latronibus antiquius esse debere quam lucrum, ne honestam quidem sed periculosam ultionem. Proinde, ne latrones ira perturbati optimam lucrandi occasionem amittant, suadet ne Charite capitis damnetur, sed tradatur potius lenoni: inde enim nonnullum quaestum latronibus proventurum, puellam vero poenam haud

praestare videtur cum doctrina tum etiam subtilitate in singulis locis rimandis expositionibus Groningianis in quibus iidem loci tractantur. 
minus acerbam passuram esse. Haec omnia mihi notissimum Sallustii locum recinere videntur, eum videlicet, in quo Caesar senatores de catilinariorum supplicio alloquitur (Sall. Cat. 51). Quodsi illam cum Tlepolemi oratione contuleris, videbis earum argumenta mirum in modum inter se congruere. Illic enim Caesar admonet senatores, ne ira occaecati rem praecipitent suppliciaque insolita de catillinariis sumere velint: timendum enim esse, nequis hoc posterius eis obiciat. Suadet itaque ut res accurate perpendatur atque in praesens catilinarii in diversa Italiae municipia relegentur: hanc enim poenam non minus duram fore quam mortem ipsam, quae aerumnarum omnium requies est.

Sed ita censeo: publicandas eorum pecunias, ipsos in vinculis habendos per municipia, quae maxime opibus valent; neu quis de iis postea ad senatum referat neve cum populo agat. Sall. Cat. 51,43

Quae verba haud multum differunt ab illo: Quin ego censeo deducendam eam ad quampiam civitatem ibique venundandam. His consideratis, haud alienum esse puto si arbitremur Apuleium hoc Sallustii loco proposito orationem Tlepolemi scripsisse - quod facile fieri poterat, cum Sallustii opera diligentissime legerentur a discipulis grammticorum rhetorumque et cum haud raro Madaurensis ipse Sallustii locos iocandi gratia Metamorphosibus iniecerit ${ }^{15}$.

Ad hoc in Tlepolemi oratione, praeter multa lumina verbaque ex curia deprompta, etiam locus communis de lucro adicitur, ubi scilicet affirmatur latronem sincerum nihil quaestui praelaturum esse. Hic orationis nostrae locus eo mirabilis est, quod vulgaria rhetorum de genere deliberativo praecepta pervertit, ut v.g. a Quintiliano in libro tertio Institutionis oratoriae exprimuntur (III 8, 2):

Nec dubito quin ii qui sunt in illa priore sententia secundum opinionem pulcherrimam ne utile quidem nisi quod honestum esset existimarint.

Latrones igitur, qui usquequaque perversis ritibus pravoque rei publicae genere utuntur, ne vinculum quidem, quod inter honestum et utile a bonis exister crediture, permiscere atque evertere dubitant: etenim, ut sapientes contendunt nihil esse utile, nisi quod et honestum, sic scelesti homines nihil honestum credunt, nisi quod et utile, hoc est, quod lucrum afferat. Itaque ultio, quae per se ipsa nonnihil ingenui honestique habet, eo ipso spernitur, quod cum quaestu repugnat.

\section{CONCLUSIO}

Haec cum sic habeant, satis liquido apparet greges latronum ita a fabularum scriptoribus Graecis tractari atque depingi, ut illorum instituta et administratio

\footnotetext{
${ }^{15}$ Cf. v.g. Finkelpearl 1998: 50.
} 
civitati cuidam legibus fundatae accedere videatur. Quin immo, latrones ipsi interdum ita sese gerunt eumque in modum loquuntur ut dignitatem verae rei publicae affectent. Apuleius autem, qui haec omnia in narrationibus Graecis reppererat, eadem ita amplificavit dictionibusque ex genere deliberativo tractis auxit ut risum lectori suscitaret: in hoc quidem tota vis artificii Apuleiani, quod his fabulis adhibetur, consistit.

Ceteroquin, ea quae hoc loco de genere deliberativo illustravimus referenda certe sunt ad illam variorum generum mixturam quae in fabulis de latronibus multifariam observamus ${ }^{16}$. Quae omnia ad memoriam revocant antiquas disputationes de parodiae natura, quam ut recte definirent atque ad mores veterum scriptorum referrent plurimi viri docti operam dederunt ${ }^{17}$. Equidem inter multas definitiones quae de hac re praebitae sunt optime in fabulas nostras convenire existimo hanc sententiam Michaelis Bachtin:

The novel parodies other genres (precisely in their role as genres); it exposes the conventionality of their forms and their language; it squeezes out some genres and incorporates others into its own peculiar structure, reformulating and re-accentuating them ${ }^{18}$.

In istis enim orationibus fictis Apuleius non certos ludificat scriptores, sed universum genus scribendi: qui lusus cum in his locis quos exhibuimus in genus deliberativum cadit, tum alibi in sermonem philosophicum, religiosum, militarem et alios. Quamquam cavendum est ne putemus Apuleium his iocis ipsa genera dicendi, quae contaminat, deprimere aut carpere voluisse: nihil est enim in Metamorphosibus neque in aliis Apuleii scriptis, ex quo suspicari possimus eum hic quicquam praeter doctum lusum afferre voluisse.

Apuleius includes and reaffirms the existence of epic's authority against his own humility, yet he clearly also subverts that authority. He has, however, incorporated and "refunctioned" epic language as part of his own narrative, and he has done so in a way that makes clear its literary nature ${ }^{19}$.

Ea quae Helena D. Finkelpearl de apuleiana parodia Vergilii atque Homeri adnotavit, eadem haud minus idonea sunt ad lusum describendum, quem Apuleius in imitatione generis deliberativi usurpat. Haec enim imitatio dum maiestatem illius generis dicendi in conspectu ponit, eodem tamen tempore nos admonet omnia quae speciose ac graviter dici solent in maximam absurditatem converti posse $a b$ artifice qui iis callide atque affabre uti sciat.

\footnotetext{
${ }^{16}$ Cf. pag. 1.

${ }^{17} \mathrm{Si}$ resciscere volueris ea quae viri docti praecipueque philologi de hoc artificio senserunt, cf. Finkelpearl 1998: 36-44.

${ }^{18}$ Bakhtin 1981: 5.

${ }^{19}$ Finkelpearl 1998: 43.
} 


\section{CONSPECTUS LIBRORUM}

Operis apvleiani editiones criticae et expositiones

Apuleio, Le metamorfosi o l'asino d'oro, introduzione, traduzione e note di L. Niccolini, Milano 2006. Apulei Metamorphoseon libri XI, recognovit brevique adnotatione critica instruxit M. Zimmeraman, Oxonii 2012.

L. Apuleii Madaurensis, Opera omnia ex optimis codicibus edidit et rerum indicem adiecit G.F. Hildebrand, Lipsiae 1843.

L. Apuleii Madaurensis, Opera quae quidem extant omnia et in primis de asino aureo libri XI, cum eruditissimis Philippi Beroaldi commentariis, Basileae 1560.

Apuleius, Metamorphoses, Book IV, 1-27. text, introd and comm. by Hijmans, Paardt, Smits, Westendorp, Westerbrink, Groningen 1977.

Symbolae, commentationes, syngrammata

Bakhtin, 1981: M.M. Bakhtin, The Dialogic Imagination, trans. Caryl Emerson and Michael Holquist, Austin 1981.

Callebat 1968: L. Callebat, Sermo Cotidianus dans le Métamorphoses d'Apulée, Caen 1968.

Facchini 2011: B. Facchini, Giurisprudenza da favola:note sul lessico giuridico delle Metamorfosi di Apuleio, "Lexis" (29) 2011, pp. 301-323.

Finkelpearl 1998: E.D. Finkelpearl, Metamorphosis of Language in Apuleius: a Study of Allusion in the Novel, Ann Arbor 1998.

Flam-Zuckermann 1970: L. Flam-Zuckermann, A propos de d'une inscription de Suisse: études sur le phenomène du brigandage, Latomus 29, 1970, pp. 451-473.

Frangoulidis 1994: S.A. Frangoulidis, Self-Imitation in Apuleius' Tales of Tlepolemus-Haemus and Thrasyleon, "Mnemosyne" 1994 (v. 45, f. 3), pp. 337-348.

Frangoulidis: S.A. Frangoulidis, Theatre and spectacle in Apuleius' tale of the robber Thrasyleon, "Griechische-römische Komödie und Tragödie" (3), pp. 113-135.

Frangoulidis 1991: S.A. Frangoulidis, Vergil's tale of Trojan Horse in Apuleius' robber-tale of Thrasyleon, "La parola del passato" 1991, pp. 95-111.

Gianotti 1981: G.F. Gianotti, Memoria letteraria e giuridica nell'episodio di Chryseros e Lamachus (Apul. met. 4,9-11), "Quaderni di Filologia Classica" 1981 III, pp. 59-83.

Grünewald 1999: T. Grünewald, Räuber, Rebellen, Rächer: Studien zu Latrones im Römischen Reich, Wiesbaden 1999.

Harrison 2013: S.J. Harrison, Framing the Ass, literary texture in Apuleius' Metamorphoses, Oxford 2013.

Harrison 2000: S.J. Harrison, Apuleius: A Latin Sophist, Oxford 2000.

Hobsbawm 1981: E. Hobsbawm, Bandits, New York 1981.

Janni 2007: P. Janni, Apuleio, i briganti e la sepoltura in mare, "Quaderni urbinati di cultura classica" 2007, pp. 125-134.

Penna 1985: A. La Penna, Una novella di Apuleio e l'Iliupersis virgiliana, "Maia" 1985, pp. $145-147$.

Lazzarini 1985: C. Lazzarini, Il modello virgiliano nel lessico delle metamorfosi di Apuleio, "Studi Classici e Orientali” 1985, pp. 131-160.

Loporcaro 1992: M. Loporcaro, Eroi screditati dal testi, "Maia" 1992, pp. 65-77.

May 2006: R. May, Apuleius and Drama, The ass on the stage, Oxford 2006.

Mazzarino 1950: A. Mazzarino, La Milesia e Apuleio, Torino 1950.

Mackay 1963, P.A. Mackay, Klephtika: The Tradition of the Tale of Banditry in Apuleius, "Greece and Rome" 1963, pp. 147-152.

McMullen 1967: R. McMullen, Enemies of the Roman Order, Treason, Unrest and Alienation in the Roman Empire, Oxford 1967. 
McMullen 1967: R. McMullen, Enemies of the Roman Order, Treason, Unrest and Alienation in the Roman Empire, Oxford 1967.

Médan 1927: P. Médan, La latinité d'Apulée dans le Métamorphoses, Paris 1927.

Nicolini 2000: L. Nicolini, La novella di Carite e Tlepolemo, Napoli 2000.

Palacios 2005: J. Palacios, Las historias de Làmaco, Alcimo y Trasyleòn, Argos 2005, pp. 91-106.

Paratore 1942: E. Paratore, La novella in Apuleio, Messina 1942.

Pasetti 2007: L. Pasetti, Plauto in Apuleio, Bologna 2007.

Perry 1923: B.E. Perry, Some Aspects of the Literary Art of Apuleius in the Metamorphoses, "Transactions and Proceedings of the American Philological Association 1923, pp. 196-222.

Rohde 1914: E.Rohde, Der Griechischer Roman und seine Verläufer, Leipzig 1914.

Scazzoso 1951: P. Scazzoso Le metamorfosi di Apuleio, studio critico sul significato del romanzo, Milano 1951.

Shaw 2006: B.D. Shaw, Il bandito, "L'uomo Romano"a cura di A. Giardina, Bari 2006.

Shaw 1984: B.D. Shaw, Bandits in the Roman Empire, Past and Present 1984, pp. 3-52.

Tatum 1979: J. Tatum, Apuleius and the Golden Ass, Ithaca and London 1979.

Van der Paardt 1978: R. Th. Van der Paardt, Various Aspects of narrative technique in Apuleius' Metamorphoses, "Aspects of Apuleius the Golden Ass", ed. Hijmans-Paardt, Groningen-Bouma 1978.

Winkler 1985: J.J. Winkler, Auctor and Actor, a Narratological Reading of Apuleius's The Golden Ass, Berkeley and Los Angeles 1985.

Westerbrink 1978: A.G. Westerbrink, Some parodies in Apuleius' Metamorphoses, "Aspects of Apuleius' the Golden Ass", ed. Hijmans-Paardt, Groningen 1978.

\section{DE GENERE DICENDI DELIBERATIVO A LATRONIBVS APULEIANIS VSVRPATO (MET. VI, VII)}

Sum mary

In greek novels we find some elements concerning the representation of the robbers' band as a sort of anti-society in which the values of the real states are partly mirrowed, partly perverted: that is, the cult of Mars conected with human sacrifice, the ethic of violence and cruelty, some strange rituals such as the see-burial, and above all the robbers' council. It is clear that this last motif had a particular relevance in the plots of greek novels: Chariton and Heliodorus have some examples of robbers' councils in which we can recognize several common themes. In these parts of their tales Chariton and Heliodorus report some robbers' speeches in which it can be found not only the greek political vocabulary of assemblies, but also an imitation of the higher style which is typical of this genre. In Apuleius' Metamorphoses all these elements are amplified and re-elaborated: in this article I tried to clarify how important is the role that they play in the comical representation of the brigands. In order to illustrate these aspects I tried to offer a rhetorical analysis of the speeches of VI 30-31 and VII 9. 